\title{
Métodos híbridos de pontos interiores e da soma ponderada aplicados ao modelo multiobjetivo de colheita de cana-de-açúcar, de coleta e geração de energia de sua biomassa.
}

\author{
Camila de Lima \\ Programa de Pós-Graduação em Ciências da Computação e Matemática Computacional , \\ ICMC, USP, CEP: 13566-590, São Carlos, SP, \\ Email: cadlima@yahoo.com.br
}

Antonio Roberto Balbo

Depto. de Matemática, FC, UNESP, CEP: 17033-360, Bauru, SP

Email: arbalbo@fc.unesp.br

Helenice de Oliveira Florentino Silva

Depto de Bioestatística, IB, UNESP, 18618-970, Botucatu, SP

Email: helenice@ibb.unesp.br

\begin{abstract}
Resumo: Nesse trabalho o modelo multiobjetivo relativo à colheita de cana-de-açúcar, à coleta e geração de energia provenientes do aproveitamento de sua biomassa é proposto a partir de outros já divulgados na literatura. Análise de soluções desse modelo é realizada explorando-se um procedimento híbrido envolvendo os métodos previsor-corretor primal-dual de pontos interiores, branch-and-bound e da soma ponderada. Este modelo visa determinar a escolha das variedades de cana-de-açúcar que minimizam o custo de colheita da cana e coleta de sua biomassa elou que maximizam o balanço de energia resultante do aproveitamento desta, considerando áreas mecanizáveis e semi-mecanizáveis, e respeitando as restrições do problema. O modelo multiobjetivo é transformado em uma classe de problemas mono-objetivo através do método da soma ponderada. $O$ método previsor-corretor primal-dual de pontos interiores é utilizado para se obter a solução ótima relaxada e o método branch-and-bound para obter a solução ótima inteira 0-1 de cada subproblema. A partir do conjunto de soluções ótimas dos subproblemas é determinado o conjunto de soluções eficientes (não dominadas) para o modelo multiobjetivo no sentido de Pareto ótimo. Os testes foram realizados em um modelo real com 10 variedades e 16 talhões através de uma implementação no software Borland $C++$ Builder 6.0 e os resultados foram comparados àqueles do aplicativo Solver do software Excel, demonstrando que o procedimento tem um bom desempenho computacional, determina o conjunto de soluções eficientes e a curva de Pareto ótimo para o modelo multiobjetivo investigado, em um tempo computacional pequeno.
\end{abstract}

\section{Introdução}

Muitos estudos têm sido propostos visando otimizar o custo de coletar e transferir a cana-deaçúcar e a biomassa residual, do campo para o centro de processamento, bem como ao uso deste resíduo para a geração de energia, considerando-se os sistemas de colheita mecanizável e semimecanizável. Nos trabalhos de [10], [3], [4], [5], [6], [10] e [12], são discutidos modelos matemáticos para a escolha de variedades de cana-de-açúcar que buscam otimizar o custo de coleta da biomassa residual e/ou a geração de energia. Em [9] e [11] são apresentados modelos de minimização de custo da colheita da cana-de-açúcar, considerando áreas mecanizáveis e semi-mecanizáveis em sua formulação.

De modo geral, o modelo multiobjetivo proposto em [6] é uma extensão daqueles divulgados nos trabalhos citados e visa minimizar o custo da colheita da cana-de-açúcar, bem como minimizar o custo de coleta e transporte da biomassa residual e/ou maximizar a geração de energia da biomassa, determinando qual tipo de variedade será plantada em cada talhão, respeitando as restrições de produção de sacarose e fibra de cana-de-açúcar, uso total da área destinada ao plantio (mecanizável ou 
semi-mecanizável) e o plantio de apenas uma variedade de cana-de-açúcar por talhão. Assim, cada variedade de cana-de-açúcar pode ou não ser plantada em determinado talhão. Este aspecto caracteriza um problema de programação inteira zero-um, e dependendo de sua dimensão, pode ser de difícil resolução. Além disso, inseriu-se uma restrição com o intuito de limitar o plantio de uma mesma variedade no conjunto de talhões.

Para a investigação de soluções desse modelo utilizou-se um procedimento híbrido envolvendo métodos previsor-corretor primal-dual de pontos interiores (MPCPDPI), branch-andbound e da soma ponderada, para a resolução de um modelo multiobjetivo referente a uma usina do estado de São Paulo, que envolve 10 variedades de cana-de-açúcar e 16 talhões para plantio. O método da soma ponderada, definido em [2], que é uma estratégia que transforma o modelo multiobjetivo em uma classe de subproblemas mono-objetivo, possibilitou o uso dos métodos híbridos de pontos interiores e branch-and-bound para a determinação de soluções desses. Foi utilizado o método previsor-corretor primal-dual de pontos interiores para se obter a solução ótima relaxada de cada subproblema e, a partir desta, determinar a solução ótima inteira relacionada às restrições de integralidade desses, relativas à escolha das variedades a serem plantadas, através do método branchand-bound. O conjunto de soluções ótimas dessa classe de subproblemas possibilitou a determinação do conjunto das soluções eficientes (não dominadas) do modelo multiobjetivo investigado e a fronteira de Pareto ótima.

\section{O Modelo Multiobjetivo}

De acordo com [9] e [11], na colheita de cana-de-açúcar queimada têm-se os custos de aceiro, queima, corte manual, carregamento da cana para o caminhão e transporte da cana do campo para a usina. Na colheita mecanizada, têm-se os custos de corte e transporte da cana do campo para a usina. Estes dados estão apresentados nas Tabelas 3 e 4 da Seção 4.

O custo de transporte da variedade $i$ plantada no talhão $j\left(C t_{i j}\right)$ a uma distância $\left(D_{j}\right)$ do talhão $j$ para a usina:

$$
C t_{i j}=c_{\text {med }_{i}} . D_{j}
$$

Em que: $i=1,2, \ldots, n$ são os índices que representam as variedades; $j=1,2, \ldots, k$ são os índices que representam os talhões; $c_{\text {med }_{i}}$ é o custo médio do transporte da cana por $\mathrm{km}$; e $D_{j}$ é a distância do talhão $j$ do centro de processamento, em talhões.

O custo $C_{i j}^{S M}$ de colheita e transporte da cana-de-açúcar de variedade $i$ plantada no talhão $j$ no sistema semi-mecanizado é calculado da seguinte forma:

$$
C_{i j}^{S M}=\left(C a_{i}+C q_{i}+C c o_{i}+C c a_{i}+C t_{i j}\right) \cdot L_{j}
$$

Em que: $C a_{i}$ é o custo de aceiro da variedade $i\left(R \$ . h a^{-1}\right) ; C q_{i}$ é o custo da queima da variedade $i\left(R \$ . h a^{-1}\right) ; C c o_{i}$ é o custo de corte da variedade $i\left(R \$ . h a^{-1}\right) ; C c a_{i}$ é o custo de carregamento da variedade $i\left(R \$ . h a^{-1}\right) ; C t_{i j}$ é o custo de transporte da variedade $i$ plantada no talhão $j\left(R \$ . h a^{-1}\right)$, calculado em (2.1); e $L_{j}$ é área do talhão $j$, em hectare.

No sistema mecanizado o custo, $C_{i j}^{M}$, de colheita e transporte da cana de variedade $i$ plantada no talhão $j$, é calculado da seguinte forma:

$$
C_{i j}^{M}=\left(C c o_{i}+C t_{i j}\right) \cdot L_{j}
$$

Em que: $C c o_{i}$ é o custo de corte da variedade $i\left(R \$ . h a^{-1}\right) ; C t_{i j}$ é o custo de transporte da variedade $i$ plantada no talhão $j\left(R \$ . h a^{-1}\right)$, calculado em (2.1); e $L_{j}$ é área do talhão $j$, em hectare.

Assim, o custo de coleta do palhiço da cana-de-açúcar da variedade $i$ plantada no talhão $j$ $\left(C C_{i j}\right)$ é calculado pela soma do custo envolvido no processo de enleirar, compactar e carregar o caminhão com o palhiço da variedade $i\left(C_{i}\right)$ e o custo de transporte do palhiço até a usina $\left(C T_{i j}\right)$, multiplicada à área do talhão $j\left(L_{j}\right)$, calculados através das Tabelas 1,2 e 5, apresentadas na Seção 4.

Abaixo, segue a expressão do custo referente ao processo de coleta dos resíduos: 


$$
C C_{i j}=\left(C_{i}+C T_{i j}\right) L_{j}
$$

A função objetivo que representa o custo total do processo de colheita da cana-de-açúcar e de coleta de resíduos, considerando áreas mecanizáveis e semi-mecanizáveis, é expresso por:

$$
C T=\sum_{i=1}^{n} \sum_{j=1}^{l}\left(C_{i j}^{M}+C C_{i j}\right) X_{i j}+\sum_{i=1}^{n} \sum_{j=l+1}^{k} C_{i j}^{S M} X_{i j}
$$

em que: $C_{i j}^{M}$ é o custo da colheita mecanizada; $C C_{i j}$ é o custo da coleta de resíduos; $C_{i j}^{S M}$ é o custo da colheita semi-mecanizada; $l$ é número de talhões em que se considera o sistema mecanizado; $k-l$ é o número de talhões em que se considera o sistema semi-mecanizado; e $X_{i j}$ são as variáveis de decisão.

Em se tratando de energia, de acordo com [3] e [4], o balanço de energia para o aproveitamento do palhiço é obtido pela diferença entre a energia proveniente do palhiço da variedade $i$ plantada no talhão $j\left(E B_{i j}\right)$ e a energia gasta na transferência palhiço da variedade $i$ plantada no talhão $j\left(E T_{B i j}\right)$ que é a soma das energias gastas para enleirar e compactar $\left(E_{E C i j}\right)$, carregar $\left(E_{C i j}\right)$ e transportar esta biomassa $\left(E_{T i j}\right)$, calculadas através dos dados das Tabelas 1,2 e 5 apresentados na Seção 4. A fórmula do balanço de energia é apresentada a seguir:

$$
B E_{i j}=E B_{i j}-E T_{B i j}
$$

Assim, define-se a função objetivo referente ao balanço de energia no aproveitamento de resíduos de cana-de-açúcar:

$$
B E T=\sum_{i=1}^{n} \sum_{j=1}^{l} B E_{i j}^{M} X_{i j}+\sum_{i=1}^{n} \sum_{j=1}^{l} B E_{i j}^{s M} X_{i j}
$$

em que: $B E T$ é o balanço de energia total no aproveitamento de resíduos da cana-de-açúcar, $B E_{i j}^{M}$ é definido de forma equivalente a (2.6) porém considera apenas áreas mecanizáveis e $B E_{i j}^{S M}$ também é calculada de forma equivalente a (2.6) levando em consideração apenas áreas semi-mecanizáveis.

Neste trabalho, os cálculos de custo de coleta e do balanço de energia referentes ao aproveitamento de resíduos das áreas semi-mecanizáveis $\left(B E_{i j}^{S M}\right)$ são considerados nulos, visto que a colheita nesta área não gera resíduos.

O modelo muitiobjetivo proposto em [7] consiste em determinar quais das variedades $i$ ( $i=1, \ldots, n)$ de cana-de-açúcar devem ser plantadas nos talhões $j(j=1, \ldots, k)$ de área $L_{j}(h a)$ e distância $D_{j}(K m)$ da usina, que investigue, simultaneamente, o mínimo custo total de colheita da cana-de-açúcar e coleta de resíduos e o máximo balanço de energia no aproveitamento de resíduos resultantes da colheita, os quais têm objetivos conflitantes, levando em consideração restrições como quantidade de produção de sacarose e fibra de cana-de-açúcar, uso total da área destinada ao plantio e o plantio de apenas uma variedade de cana-de-açúcar por talhão. O modelo matemático é definido por:

$$
\begin{array}{cc}
\text { Minimizar } & (C T ;(-1) B E T) \\
\text { Sujeito } a: & \sum_{i=1}^{n} \sum_{j=1}^{k} A_{i} X_{i j} \geq \bar{A} T ; \\
& \overline{F_{l}} T \leq \sum_{i=1}^{n} \sum_{j=1}^{k} F_{i} X_{i j} \leq \overline{F_{S}} T ; \\
& \sum_{i=1}^{n} X_{i j}=1 ; \\
X_{i j}=0 \text { ou } 1, i=1,2, \ldots, n \text { e } j=1,2, \ldots, k \\
\sum_{j=1}^{k} X_{i j} \leq M
\end{array}
$$

Além de $C T$ e e $B E T$, as quais estão definidas em (2.5) e (2.7); $i=1,2, \ldots, n$ são os índices que representam as variedades, $j=1,2, \ldots, k$ são os índices que representam os talhões; $A_{i}$ é a estimativa de produção de sacarose da variedade $i(t / h a) ; \bar{P}$ é a quantidade mínima estabelecida para a POL da cana; $T$ é o número total de talhões; $F_{i}$ é a estimativa do teor de fibra da variedade $i ; \overline{F_{I}}$ e $\overline{F_{S}}$ são as quantidades mínimas e máximas estabelecidas para a fibra da cana; $M$ é o número máximo que cada variedade $i$ pode ser plantada.; $X_{i j}=1$ implica que a cana de variedade $i$ deve ser plantada no talhão $j$ e em caso contrário $X_{i j}=0$. 


\subsection{Estratégias de resolução}

Geralmente, os modelos multiobjetivo são de difícil resolução e na maioria das vezes exigem a intervenção do usuário para a determinação de soluções satisfatórias (eficientes). Assim, para a resolução deste modelo multiobjetivo, pode ser utilizada a estratégia de otimização conhecida por Otimalidade de Pareto, através dos métodos da soma-ponderada relacionado ao modelo (2.10) e do $\varepsilon$ restrito, relacionado ao modelo (2.11), baseando-se em [2]. Esses métodos transformam o modelo em uma classe de subproblemas mono-objetivos, buscam determinar o conjunto de soluções eficientes para o modelo e possiblitam a construção da curva de Pareto ótimo. Nos testes realizados apenas o modelo (2.10) foi mostrado, os resultados para o modelo (2.11) podem ser vistos em [7].

\section{Método da soma ponderada}

Minimizar $\quad(\alpha C T-(1-\alpha) B E T)$

Sujeito a: $\quad$ Restrições (2.9)

em que $0 \leq \alpha \leq 1$.

\section{Método do $\varepsilon$-restrito}

Maximizar BET

Sujeito a: Restrições (2.9) e

$C T \leq \varepsilon$

\section{Método previsor-corretor primal-dual de pontos interiores e branch-and-bound}

O método primal-dual com procedimento previsor-corretor utilizado é variante daquele proposto por [8] diferenciando-se deste por já utilizar no passo previsor informações do parâmetro de barreira $\mu_{k}$, o que melhora a eficiência do método por evitar que os pontos definidos por este, aproximem-se da fronteira do problema, podendo, inclusive, inviabilizá-los. Enquanto que, no passo corretor, este reajusta as direções com informações dos aproximantes de segunda ordem referentes às condições de complementaridade, possibilitando que, o procedimento de centragem do passo previsor mais o ajuste feito no passo corretor, acelerem a convergência do processo, para a determinação da solução ótima do problema contínuo.

Além disso, os modelos investigados, relativos ao processo envolvido na colheita da cana-deaçúcar e ao aproveitamento de sua biomassa residual, consistem em determinar se uma variedade é plantada ou não. Este problema enquadra-se como um problema de programação inteira binária (PIB) ou programação inteira zero-um, aquele cujas variáveis podem assumir valores 0 ou 1 . A busca de soluções para este tipo de problema, com grande número de variáveis e restrições, é de difícil resolução e nem sempre o sucesso na determinação de soluções é garantido, principalmente em casos multiobjetivos. No procedimento híbrido proposto, o método de programação inteira utilizado foi o método branch-and-bound, baseando-se em [1].

O desenvolvimento do método, bem como todos os passos do Algoritmo previsor-corretor primal-dual e branch-and-bound (PDBB) podem ser vistos em [7], já que, nesse texto a limitação do número de páginas impossibilitou o detalhamento do passos desse método, os quais incluíram, no Procedimento externo (resolução do PIB relaxado): um teste de otimalidade que incluiu o teste de integralidade de Borches e Mitchell [1]; os cálculos intermediários do passo previsor: os cálculos intermediários do passo corretor (que envolve a atualização dos termos de segunda ordem das folgas complementares); as direções de busca determinadas no passo corretor; teste de ilimitariedade; comprimento dos passos primal e dual e atualização da solução. No procedimento interno a execução dos passos do método Branch-and-Bound (avaliação do problema, ramificação, atualização da solução e seleção do novo nó a ser investigado), utilizando o MPCPDPI para a atualização e integralização das soluções.

\section{Resultados}

Para a obtenção de resultados, o algoritmo previsor-corretor primal-dual e branch-and-bound (PDBB), visto na Seção 3, foi implementado no software Borland C++ Builder 6.0. Assim, foi possível fazer a aplicação do método PDBB ao modelo multiobjetivo investigado referente à minimização dos custos de colheita de cana-de-açúcar e coleta de resíduos, e à maximização de energia relativa ao 
aproveitamento de resíduos, apresentado na Seção 2.2, para obter o conjunto de soluções eficientes desse modelo. Com o intuito de validar estas soluções, os resultados obtidos foram comparados com aqueles obtidos pelo aplicativo Solver do software Excel.

Para os cálculos referentes ao modelo multiobjetivo investigado, utilizou-se os dados das Tabelas de 1 a 5, apresentadas em [6], [9] e [11]. A Tabela 1 apresenta as estimativas por tipo de variedades, considerando 10 variedades. A Tabela 2 apresenta os custos, consumos, e recomendações referentes às variedades. A Tabela 3 apresenta os custos referentes ao processo de transporte. A Tabela 4 apresenta os custos referentes ao processo de colheita. E por fim, a Tabela 5 apresenta a área e a distância dos talhões à usina, considerando 16 talhões.

TABELA 1: Estimativas de valores por variedade.

\begin{tabular}{ccccccccc}
\hline$i$ & Variedade & $V i$ & $P_{B i}$ & $E_{C B i}$ & $A i$ & $Q i$ & $F i$ & $P c$ \\
\hline 1 & SP80-1816 & 7,964 & 33,360 & 2671,990 & 16,420 & 354,200 & 13,940 & 100,000 \\
2 & RB72454 & 8,610 & 37,580 & 2649,950 & 20,400 & 299,280 & 12,900 & 186,000 \\
3 & SP80-3280 & 9,369 & 36,720 & 2602,140 & 18,460 & 316,180 & 12,630 & 158,000 \\
4 & SP81-3250 & 10,619 & 34,250 & 1947,850 & 18,380 & 320,850 & 11,320 & 179,000 \\
5 & RB855536 & 9,780 & 26,430 & 2211,950 & 17,050 & 258,460 & 12,510 & 165,000 \\
6 & RB855113 & 10,870 & 29,380 & 2310,370 & 17,540 & 319,380 & 10,910 & 155,000 \\
7 & SP79-1011 & 8,910 & 24,090 & 1977,470 & 15,800 & 214,720 & 10,330 & 158,000 \\
8 & RB835486 & 9,560 & 21,530 & 2444,200 & 12,840 & 205,770 & 9,280 & 155,000 \\
9 & RB711406 & 12,320 & 33,200 & 2008,830 & 20,770 & 410,290 & 16,120 & 183,000 \\
10 & SP70-1143 & 7,050 & 22,140 & 1924,800 & 15,010 & 155,980 & 11,590 & 155,000 \\
\hline
\end{tabular}

TABELA 2: Custos e consumos de combustível e energia dos maquinários utilizados para a coleta do palhiço e recomendações de teores de Pol e fibra da cana-de-açúcar.

\begin{tabular}{cccccccccc}
\hline$C e_{c c}$ & $C o$ & $P$ & $V_{c}$ & $E c_{E C}$ & $E c_{c}$ & $E c_{T}$ & $\bar{A}$ & $\bar{F}_{I}$ & $\bar{F}_{S}$ \\
\hline 3,69 & 0,37 & 2,15 & 7,56 & 57,54 & 5,25 & 60 & 14 & 11 & 15 \\
\hline
\end{tabular}

TABELA 3: Custos envolvidos no processo de transporte.

\begin{tabular}{cc}
\hline Transporte & Custo \\
\hline Cana crua & 6,42 \\
Cana queimada & 5,35 \\
\hline
\end{tabular}

TABELA 4: Custos envolvidos no processo de colheita.

\begin{tabular}{lc}
\hline \multicolumn{1}{c}{ Operação } & Custo \\
\hline Aceiro & 0,14 \\
Queima & 0,17 \\
Corte & 7,03 \\
Corte cana crua & 10,5 \\
Carregamento & 1,62 \\
\hline
\end{tabular}

TABELA 5: Área e distância dos talhões até a usina.

\begin{tabular}{c|cccccccc}
\hline Talhão $j$ & 1 & 2 & 3 & 4 & 5 & 6 & 7 & 8 \\
\hline$L_{j}$ & 8,490 & 4,520 & 58,180 & 4,220 & 5,740 & 6,610 & 30,410 & 5,080 \\
$D_{j}$ & 3,490 & 2,490 & 16,080 & 3,490 & 2,590 & 2,590 & 15,330 & 8,300 \\
\hline Talhão $j$ & 9 & 10 & 11 & 12 & 13 & 14 & 15 & 16 \\
\hline$L_{j}$ & 12,010 & 54,950 & 38,660 & 3,780 & 10,430 & 6,150 & 8,790 & 57,790 \\
$D_{j}$ & 9,240 & 12,630 & 16,430 & 8,250 & 7,800 & 8,590 & 2,250 & 17,200 \\
\hline
\end{tabular}

E ainda, para a aplicação do método PDBB ao modelo apresentado na Seção 2.2, foram definidos os talhões 3 e 11 para áreas semi-mecanizáveis, e os demais para as áreas mecanizáveis.

\subsection{Análise de resultados}

Encontrar uma solução ótima para o modelo multiobjetivo não é possível, visto que os objetivos de minimizar o custo total e maximizar o balanço de energia total da colheita da cana-deaçúcar, coleta e aproveitamento dos resíduos são conflitantes. Desta forma, utilizam-se as estratégias da soma ponderada e do $\varepsilon$-restrito, definido pelos problemas (2.10) e (2.11), respectivamente, que transformam o modelo multiobjetivo (2.8) em um conjunto de problemas mono-objetivo. Assim, a partir da aplicação do método PDBB, visto na Seção 3, a estes problemas, pode-se obter um conjunto 
de soluções eficientes, as quais determinam a curva de Pareto. Nesse trabalho apresentamos os resultados apenas para os problemas (2.10). Os resultados obtidos para (2.11) podem ser vistos em [7].

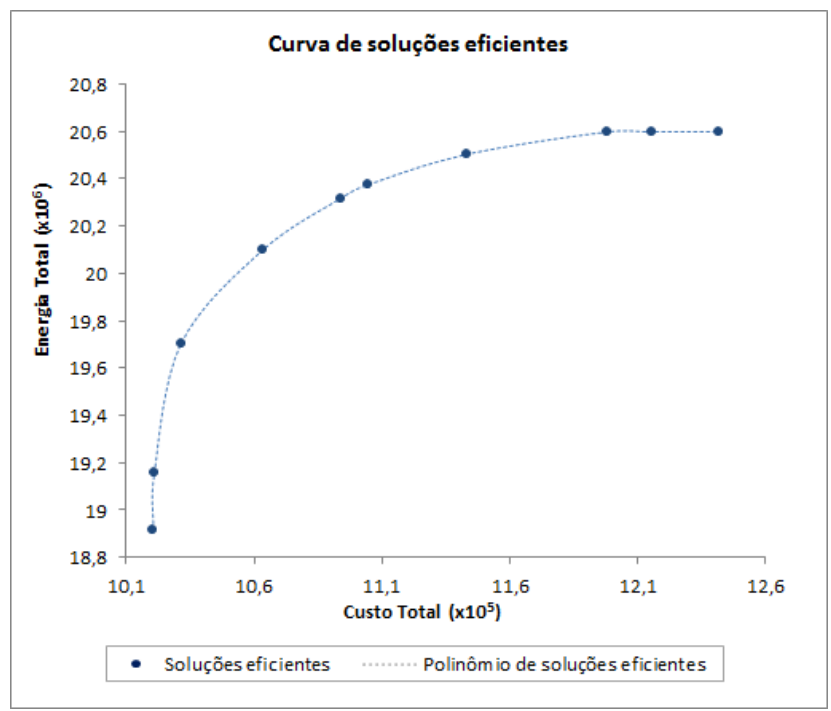

TABELA 6: Valores das funções objetivo obtidos pelo método PDBB a partir da estratégia da soma ponderada.

\begin{tabular}{ccc}
\hline Valor de & Custo total & Energia total \\
$\alpha$ & $C T$ & $B E T$ \\
\hline 0 & $1.241 .777,91$ & $20.597 .468,47$ \\
0,055 & $1.215 .504,75$ & $20.597 .468,47$ \\
0,065 & $1.198 .046,52$ & $20.597 .468,47$ \\
0,08 & $1.143 .057,66$ & $20.502 .583,59$ \\
0,18 & $1.104 .590,43$ & $20.374 .393,59$ \\
0,2 & $1.093 .947,18$ & $20.317 .323,13$ \\
0,3 & $1.063 .298,50$ & $20.098 .430,45$ \\
0,45 & $1.031 .361,26$ & $19.703 .458,44$ \\
0,87 & $1.020 .911,42$ & $19.155 .067,67$ \\
1 & $1.020 .556,80$ & $18.915 .734,85$ \\
\hline
\end{tabular}

FIGURA 1: Curva de soluções eficientes obtida pelo método PDBB a partir da estratégia da soma ponderada.

A Figura 1 apresenta a relação entre os valores de custo e balanço de energia total, e o fato destes objetivos serem conflitantes, melhorar um objetivo, implica em piorar o outro. As soluções eficientes obtidas são de interesse, pois informam ao produtor a determinação das variedades a serem plantadas, de modo a atender o momento econômico de sua empresa. Ainda, de acordo com Lima (2013), a aplicação do método PDBB à estratégia do ع-restrito gera um número maior de soluções eficientes do que a estratégia da soma ponderada, visto que esta estratégia permite que o usuário escolha o limitante $\varepsilon$, de acordo com suas necessidades.

\section{Conclusões}

Neste trabalho, foi proposto um modelo multiobjetivo para minimizar o custo da colheita da cana-de-açúcar e o custo da coleta da biomassa residual, e/ou maximizar o balanço de energia relativo à biomassa residual do processo, baseando-se em Ramos (2010), Silva (2011), Florentino (2006), Florentino et al (2011), Homem (2010) e Homem et al (2011). Este foi investigado através do método híbrido PDBB e da soma ponderada para um problema real de uma usina do estado de São Paulo contendo dezesseis talhões e dez variedades, em que as áreas dos talhões 3 e 11 foram consideradas semi-mecanizáveis e as demais, mecanizáveis.

O algoritmo do método PDBB foi implementado através do software Borland C++ Builder 6.0, e aplicado ao problema destacados, através da estratégia da soma ponderada, obtendo as soluções ótimas para os casos considerados, as quais possibilitaram a determinação do conjunto de Pareto ótimo para o problema multiobjetivo testado. As soluções obtidas foram comparadas com aquelas determinadas pelo aplicativo Solver, do software Excel, e os resultados obtidos, revelaram o bom desempenho do método híbrido proposto.

$\mathrm{O}$ trabalho atingiu os seus objetivos, relacionados à proposta do modelo e ao uso do método PDBB e da soam ponderada à resolução do modelo de cultivo e de aproveitamento da biomassa residual da cana-de-açúcar, mostrando a viabilidade de se utilizar esta técnica de otimização para auxiliar as usinas na seleção de variedades a serem plantadas, de tal forma a otimizar o processo, respeitando-se as restrições de produção caracterizadas no modelo.

\section{Agradecimentos}

À CAPES pela bolsa de mestrado concedida ao $1^{\circ}$. autor. 


\section{Referências Bibliográficas}

[1] B. Borches, J. E. Mitchell, "Using an interior point method in a branch and bound algorithm for integer programming". Technical Report 195, Mathematical Sciences, Resselaer Polytechnic Institute, Troy, NY 12180, March 1991, Revised July 7, 1992.

[2] K. Deb, "Multi-objective optimization using evolutionary algorithms". John-Willey \& Sons Ltda., 2004.

[3] Florentino, H. O., "Programação linear inteira em problemas de aproveitamento da biomassa residual de colheita da cana-de-açúcar". Tese (Livre Docência) Instituto de Biociências de Botucatu, Universidade Estadual Paulista, Botucatu, SP, 2006.

[4] H. O. Florentino, A. D. Lima, L. Carvalho, A. R. Balbo, T. P. D. Homem, Multiobjective 0-1 integer programming for the use of sugarcane residual biomass in energy cogeneration. International Transactions in Operational Research, v.18, p. 605-615, (2011).

[5] T. P. D. Homem, A. R. Balbo, H. O. Florentino, Optimal energy generation with biomass of sugarcane harvest. Revista IEEE América Latina, v.1, p. 653-658, (2011).

[6] A. D. Lima, "Otimização do aproveitamento do palhiço de cana-de-açúcar". Tese (Doutorado em Energia na Agricultura), Faculdade de Ciências Agronômicas, Universidade Estadual Paulista, Botucatu, SP, 2009.

[7] C. Lima, "Métodos Híbridos de Pontos Interiores e de Programação Inteira 0-1 para Problemas de Custo de Colheita da Cana-de-Açúcar e de Custo de Coleta e Geração de Energia Relacionados à sua Biomassa". Dissertação de Mestrado, Programa de Pós-Graduação em Engenharia Elétrica - FEB, Unesp de Bauru, 2013.

[8] S. Mehrotra, On the implementation of a primal-dual interior point method. SIAM Journal on Optimization, v.2, p. 572-601, (1992).

[9] R. P. Ramos, "Modelo matemático para custo e energia na produção de açúcar e álcool". Dissertação (Mestrado em Agronomia/Energia na Agricultura), Faculdade de Ciências Agronômicas, Universidade Estadual Paulista. Botucatu, 2010.

[10] T. C. C. Ripoli, M. L. C. Ripoli, "Biomassa de cana-de-açúcar: colheita, energia e ambiente". Publicações Técnicas, ESALQ-USP, Piracicaba SP, 2004.

[11] L. M. Silva, "Algoritmo Genético na Otimização do Custo de Colheita e de Transporte da Canade-Açúcar". Dissertação (Mestrado em Biometria), Faculdade de Ciências Agronômicas, Universidade Estadual Paulista. Botucatu, 2011.

[12] G. Tolentino, "Programação Linear Inteira Aplicada ao Aproveitamento do Palhiço da Cana-deAçúcar". Dissertação (Mestrado em Agronomia/Energia na Agricultura), Faculdade de Ciências Agronômicas, Universidade Estadual Paulista, Botucatu, SP, 2007. 